\title{
Effect of the methanolic extract of Brachylaena discolor in a streptozotocin-induced diabetic rat model
}

\author{
J. J. Mellem ${ }^{1^{\star}}$, H. Baijnath ${ }^{1,2}$ and B. Odhav ${ }^{1}$ \\ ${ }^{1}$ Department of Biotechnology and Food Technology, Durban University of Technology, ML Sultan Campus, \\ Durban, 4000, South Africa. \\ ${ }^{2}$ School of Life Sciences, University of KwaZulu-Natal, Westville Campus, Durban, 4000, South Africa.
}

Accepted 11 February, 2013

\begin{abstract}
Traditionally, leaf infusions of Brachylaena discolor are used for the treatment of diabetes and renal conditions. Therefore, the aim of this study was to evaluate the effect of a methanolic leaf extract of $B$. discolor in a streptozotocin (STZ)-induced diabetic rat model. Diabetes was induced in Wistar rats by an intraperitoneal injection of STZ; blood glucose levels greater than $20 \mathrm{mmol} / \mathrm{L}$ measured after 7 days confirmed a stable diabetic mellitus state. Two doses of the test extract $(50$ and $150 \mathrm{mg} / \mathrm{ml})$ were administered daily via oral dosing to both STZ-induced and control rats. Blood was obtained from the tail vein and used to measure the effects of the extract on the biochemical profile of the rats over 28 days. The methanolic leaf extract of $B$. discolor at both doses (50 and $150 \mathrm{mg} / \mathrm{ml}$ ) caused a significant reduction in the blood glucose levels at 7, 14, 21 and 28 days in STZ-induced diabetic rats when compared with the normal rats (negative control). Significant differences were also observed in alkaline phosphatase, creatinine, total bilirubin and body weights of extract treated diabetic rats when compared with untreated diabetic rats, normal rats and metformin treated rats. Findings suggest that $B$. discolor exhibits significant anti-hyperglycaemic activity in STZ-induced diabetic rats.
\end{abstract}

Key words: Diabetes, streptozotocin, Brachylaena discolor, Wistar rats.

\section{INTRODUCTION}

Diabetes mellitus (DM) is a metabolic disease which occurs as a result of insulin deficiency and/or insulin resistance and is a major cause of disability and hospitalization (Kamgang et al., 2008). Diabetes mellitus has become a major health concern in the world, with an expectation that diabetes and obesity will reach epidemic proportions, affecting third world developing countries to a far greater extent than the developed world (Rheeder, 2006). This may be attributed to the high number of African countries, which are undergoing a demographic transition and coming under increased western influence.
This has resulted in an increase in the consumption of fat, salt and sugar, coupled with a rapid increase in urbanization in African countries and increase in nutritional deficiency, thereby providing an elevated risk factor for the prevalence of diabetes (Colagiuri et al., 2006). Estimates from the World Health Organization (WHO) have projected an increase from 171 million in the year 2000 to 366 million in 2030, with approximately $70 \%$ occurring in developing countries (WHO, 2002; Wild et al., 2004). Currently, type 2 diabetes is the most prevalent form of diabetes mellitus; however, oral 
hypoglycemic drugs which are the main form of treatment, have been shown to have undesirable sideeffects and high secondary failure rates (Moller, 2001). In addition to the lack of efficacy by pharmacological agents for the treatment of type 2 diabetes, there is also limited access to these drugs for people living in many rural areas as well as cost implications (Holt, 2004; Tonye Mahop and Mayet, 2007). These limitations have prompted research into investigating traditional medicines as potential replacements for conventional pharmacological agents used in the treatment of diabetes (Grover et al., 2002; Odhav et al., 2010). There are already an estimated 122 drugs from 94 plant species, which have been discovered through ethnobotanical leads such as indigenous knowledge. Plants such as Syzygium cordatum, Allium sativum and Ficus thonningi prevail as the treatment for diabetes in poor socio-economic conditions (Musabayane et al., 2005). These plants have been found to control diabetes mellitus by exhibiting hypoglycaemic and antioxidant effects. These ethnobotanical studies are promoted by the WHO for the discovery of novel mechanisms for diabetes treatment (WHO, 1999). Currently, in South Africa, there are no regulations with regard to the prescription and use of traditional medicine, exposing people to misadministration, especially of toxicity of plant compounds. The potential genotoxic effects that follow prolonged use of some of the more popular herbal remedies, are also cause for alarm (Fennell et al., 2004). Therefore, it becomes necessary to identify the amylase inhibitors from natural sources having lesser side-effects. The traditional African herbal medicinal system practiced for thousands of years have reports of anti-diabetic plants with no known side effects. Such plants and their products have been widely pre-scribed for diabetic treatment all around the world with less known mechanistic basis of their functioning. Therefore, these natural products need to be evaluated scientifically in order to confirm claims for their anti-diabetic properties (Iwueke and Nwodo, 2008). The aim of this study was to investigate Brachylaena discolor var. discolor as a possible dietary adjunct or therapeutic for diabetes therapy. $B$. discolor is a species of tree belonging to Asteraceae family. There are three known varieties: var. discolor which is commonly known as the Coast Silver Oak or Coastal Silver Oak, var. transvaalensis commonly known as the Forest Silver Oak or Natal Silver Oak and var. rotundata. B. discolor var. discolor is an evergreen shrub or small tree usually 4 to $10 \mathrm{~m}$ in height which is often multi-stemmed. It is confined mainly to the coastal bush and associated bushveld (van Wyk and van Wyk, 2007). These trees are distributed from the Eastern Cape in South Africa and into Southern Mozambique, but are most common in the coastal vegetation of KwaZuluNatal. The leaf infusions of $B$. discolor var. discolor are used for diabetes and renal conditions by different ethnic groups in Southern Africa and are reported to act as a tonic (Hutchings, 1996). The roots are used to treat roundworms and chest pains.

\section{MATERIALS AND METHODS}

\section{Plant}

Leaves of B. discolor var. discolor was collected in Durban, province of Kwazulu Natal, South Africa, and identified by using available floral keys. The leaves were carefully examined for insectdamage or fungus-infection and these were discarded. Healthy leaves were dried at $40^{\circ} \mathrm{C}$ for $48 \mathrm{~h}$ in a convection oven. Once completely dry, plant was ground to a fine powder using a Waring blender. The dried plant was then stored in Amber Schott bottles at room temperature until required. The methanolic extract of the dried plant material was prepared according to the procedure outlined by Jeremy and Whiteman (2003) with minor modifications. $20 \mathrm{~g}$ of the dried plant material was stirred for $24 \mathrm{~h}$ in $200 \mathrm{ml}$ of $80 \%$ methanol. The slurry was filtered using Whatman No. 1 filter paper and the supernatant was collected. The supernatant was concentrated using a Buchi RE Rotoevaporator including a Buchi 461 water bath set at a temperature of $45^{\circ} \mathrm{C}$. The concentrate was placed in a biofreezer and freeze-dried using a Virtis Benchtop Freeze Dryer. The freeze-dried material was used as a stock and working solutions were prepared for appropriate applications.

\section{Animals}

Male Wistar rats of 250 to $300 \mathrm{~g}$ body weight (BW) were obtained from the Biomedical Resource Centre (BRC) at the University of KwaZulu-Natal and divided into non-diabetic and diabetic groups of $\mathrm{n}=12$ to be used for acute and chronic studies. These animals were maintained under laboratory conditions of temperature $\left(22\right.$ to $\left.24^{\circ} \mathrm{C}\right)$, humidity (40 to $60 \%$ ) and $12 \mathrm{~h}$ light/12 $\mathrm{h}$ dark regime at the Biomedical Resource Unit (University of Kwazulu Natal, Westville Campus) where animals were exposed to both food and water ad libitum for the entire duration of the study. All animals used for this study were maintained according to the rules and regulations outlined by the Ethics Committee of the University of KwaZuluNatal, South Africa (Ethics approval was obtained from the University of KwaZulu Natal, Ethics reference 089/11/Animal).

\section{Experimental design}

The two groups of animals were sub-divided into a control group, metformin group ( $500 \mathrm{mg} / \mathrm{kg} \mathrm{BW}$ ) and treated group, each with 12 rats per group. These animals were administered two different concentrations of the crude plant extract (200 and $600 \mathrm{mg} / \mathrm{kg} \mathrm{BW}$ ) reconstituted in $1 \%$ Tween 80 and standard treatments through feeding $1 \mathrm{ml}$ of the plant extract (gavage) and standard treatment to alleviate any strain on the animal during dosage. The crude extract and standard treatment were administered to the animals for 28 days after identification of streptozotocin (STZ)-induced rats. All blood samples were obtained from the tail vein every 7 days. The study also involved a Humane Endpoint for the experiment and was conducted by veterinary support staff on a daily basis. This was used to determine the severity of the animals' well-being for the duration of the study through visual and intrusive tests (blood glucose levels, weight loss, dehydration etc).

\section{Induction of diabetes}

Diabetes was induced in male Wistar rats of 250 to $300 \mathrm{~g} \mathrm{BW}$ by 
intraperitoneal injection of STZ (60 mg/kg BW) in citrate buffer, at a pH of 4.5 (Musabayane et al., 2005). The maximum quantity for the STZ injection used was $1.0 \mathrm{ml} / 250 \mathrm{~g}$ body mass. Blood was collected via a tail prick after $48 \mathrm{~h}$ and tested using a Glucocheck blood glucose monitoring system. Animals that exhibited blood glucose levels greater than $20 \mathrm{mmol} / \mathrm{L}$ after $48 \mathrm{~h}$ were considered to be diabetic. Plasma glucose levels of $20 \mathrm{mmol} / \mathrm{L}$ measured after 7 days confirmed a stable diabetic mellitus state.

\section{Serum biochemical analysis}

For serum biochemical tests rats were warmed in a warming cabinet for 10 to $15 \mathrm{~min}$ in order to dilate blood vessels prior to taking the sample. During the warming process, rats were monitored for signs of hyperthermia and dehydration. After warming, the tail of the rat was washed with diluted Hibitane in order to see the blood vessel. Rats were restrained for the minimum time while the blood was being drawn from the lateral tail vein at the base of the tail. Approximately $1 \mathrm{ml}$ of blood was collected from 3 rats in each of the respective group every 7 days. After tail bleeding, blood flow was stopped by applying finger pressure to the blood sampling site for approximately $30 \mathrm{~s}$ before the rat was returned to its cage. Blood was centrifuged and the serum was collected and analyzed immediately using a Chemvet analyzer for the evaluation of serum biochemical parameters. The parameters measured were albumin (ALB), alkaline phosphatase (ALKP), alanine amino transferase (ALT), amylase, blood urea nitrogen (Urea), calcium $(\mathrm{Ca})$, cholesterol $(\mathrm{CHOL})$, creatinine (CREA), globulin (GLOB), phosphorous (P), total bilirubin (TBIL), total protein (TP) and blood glucose (GLU).

\section{Measurement of BW}

All experimental animals were weighed every 7 days for the entire 28 day treatment period, to monitor any weight losses or gains as a result of extract dose.

\section{Statistical analysis}

Analysis was carried out using one-way analysis of variance (ANOVA; GraphPad Prism), followed by Tukey test for multiple comparisons. Values are expressed as a mean \pm standard deviation $(n=3)$. A P-value less than 0.05 was considered to be statistically significant.

\section{RESULTS}

The result of the effect of $B$. discolor methanolic extract on the blood glucose levels of STZ-induced diabetic rats are as shown in Figure 2. These results show that nondiabetic rats (Groups 5 to 8 ) had blood glucose levels that fell within the normal range. The methanolic leaf extract of $B$. discolor in both doses $(200$ and $600 \mathrm{mg} / \mathrm{kg}$ $\mathrm{BW})$, the reference drug used (metformin) caused a time dependant $(P<0.001)$ reduction in the blood glucose levels of the STZ-induced diabetic rats (Groups 1 to 3 ) when compared with the negative control (Group 4). There was also a variation between the different doses tested on the blood glucose levels with the higher dose $(600 \mathrm{mg} / \mathrm{kg} \mathrm{BW})$ giving the highest activity followed by the reference drug metformin.
The activity of ALKP in diabetic treated rats is as shown in Figure 1. Rats treated with $B$. discolor extracts and the reference drug, decreased the ALKP levels in comparison to the untreated diabetic rats. However, the lower dose extract (200 mg/kg BW) decreased the ALKP levels within the acceptable range when compared with the higher dose and reference drug.

Creatinine levels as shown in Figure 1 shows that the diabetic rats (Group 4) exhibited a CREA level below the acceptable limits; however, animals that received both doses of $B$. discolor (Groups 1 and 2) extract and the reference drug (Group 3) increased the CREA levels to an acceptable limit. Urea levels also varied with the STZinduced control group (Group 4) and the group receiving the reference drug (Group 3) having elevated urea levels, which exceeded the reference range at the end of the 28 days treatment period.

There was a significant difference in TBIL levels between the different groups as reflected in Figure 2. Diabetic rats which received the $B$. discolor extracts (Groups 1 and 2), showed TBIL levels within the normal range when compared with the diabetic negative control (Group 4) and reference drug (Group 3).

The levels between the different treatment groups for ALB, ALT, Ca, CHOL, GLOB, P, TBIL and TP were not significant, with only small variations in levels.

The data for BW change is represented in Figure 3. Although, there was no significant difference in the initial BW (Day 0) between the different groups, the BW gains for the diabetic negative control (Group 4) and the STZinduced rats which received the low $B$. discolor dose (200 $\mathrm{mg} / \mathrm{kg}$ BW, Group 1) were significantly lower when compared with the other groups. It is important to note that the STZ-induced rats which received the higher $B$. discolor dose (Group 3) had a better BW gain in comparison to those STZ-induced rats which received the reference drug (Group 4).

\section{DISCUSSION}

There are currently no regulations controlling the prescription and use of traditional medicine in South Africa resulting in possible misadministration, especially of toxic plants. The potential genotoxic effects that follow prolonged use of some of the more popular herbal remedies, are also cause for alarm (Fennell et al., 2004). Therefore, it becomes necessary to identify the amylase inhibitors from natural sources having lesser side-effects. The purpose of this study was to investigate the antidiabetic activity of a methanolic extract of $B$. discolor in a STZ-induced diabetic rat model. This was achieved by monitoring the blood glucose levels over a 28 day period using a Chemvet analyzer. Results from the study show that the methanolic extract of $B$. discolor at the test doses and the reference drug (metformin) exhibited a significant time dependant decrease in blood glucose levels of the STZ-induced diabetic rats with different levels of 

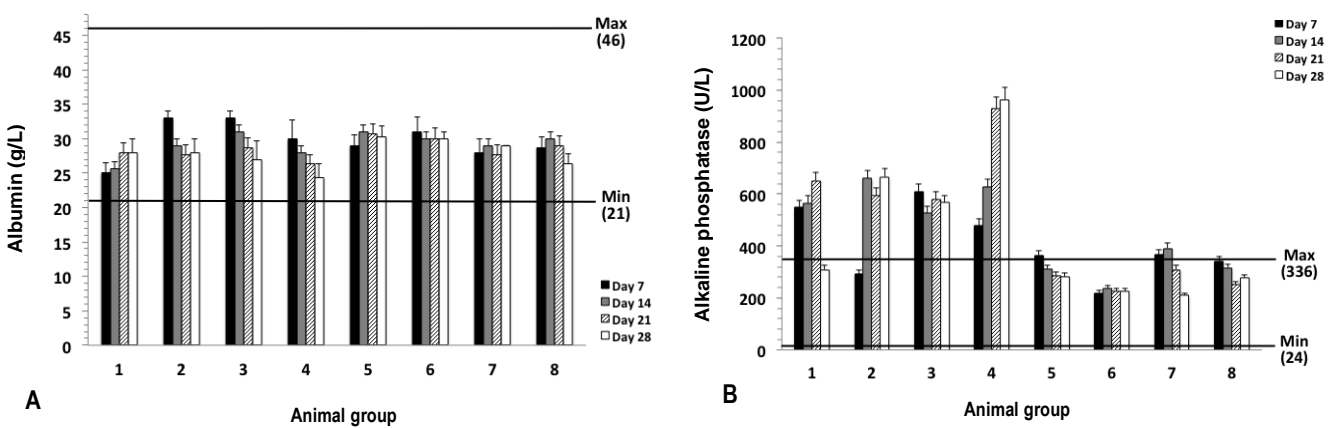

$$
\text { A }
$$

Animal group
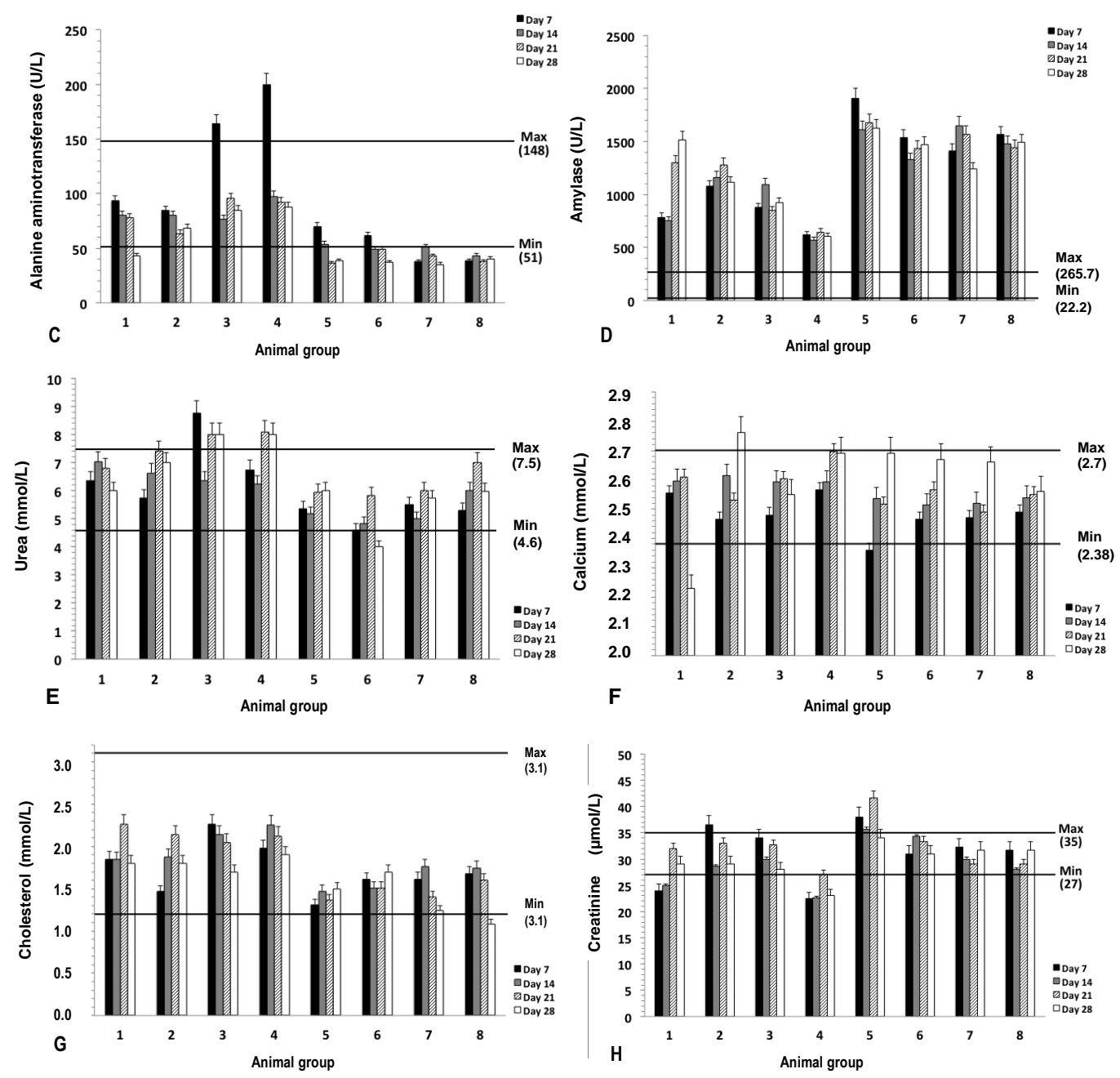

Figure 1. Effect of oral administration of $B$. discolor crude methanolic extract at doses of 200 (Ext 1 ) and $150 \mathrm{mg} / \mathrm{kg} \mathrm{BW}($ Ext 2) BW on albumin (A), alkaline phosphatase (B), alanine amino transferase $(C)$, amylase $(D)$, blood urea nitrogen $(E)$, calcium $(F)$, cholesterol $(G)$ and creatinine $(H)$ levels in normal and diabetic male Wistar rats. Each column represents mean $\pm S D(n=3)$. Group 1: Diabetic + Ext 1; Group 2: diabetic + Ext 2; Group 3: diabetic + metformin; Group 4: diabetic control; Group 5: normal + Ext 1; Group 6: normal + Ext 2; Group 7: normal control; Group 8: normal + 1\% Tween 80.

reduction on day 28 when compared with the negative control rats. In this experiment, the extract dose of 600 $\mathrm{mg} / \mathrm{kg}$ BW produced the highest antidiabetic effect and suggests that this dose may be an effective antidiabetic dose for the crude $B$. discolor extract. The variation between the 200 and $600 \mathrm{mg} / \mathrm{kg}$ BW doses may be due to the concentrations of phytochemicals and active compounds within the extract. The exact mechanism by which $B$. discolor reduces blood glucose levels are unclear, but some attribute the anti-hyperglycaemic 

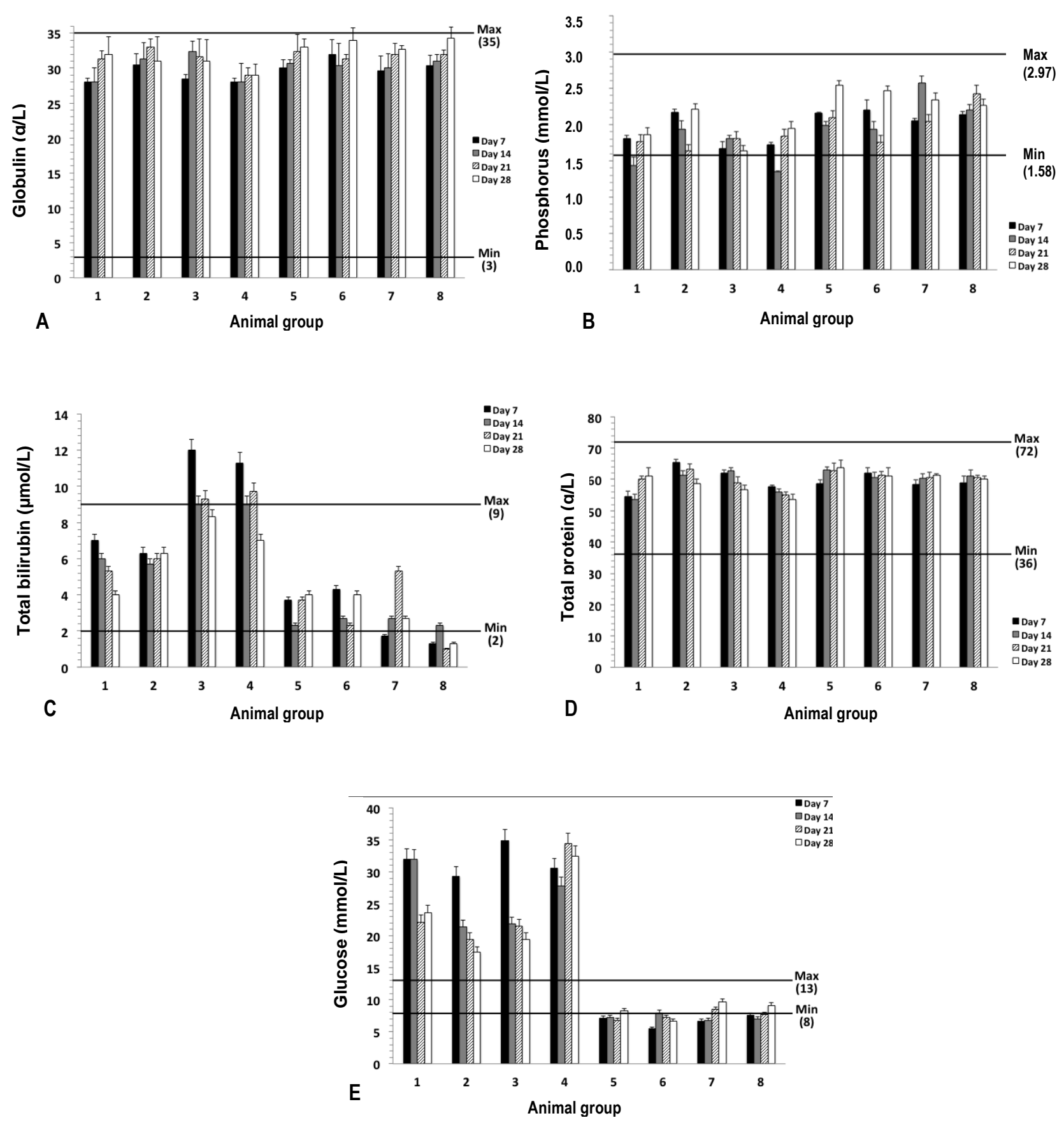

Figure 2. Effect of oral administration of $B$. discolor crude methanolic extract at doses of 50 (Ext 1) and $150 \mathrm{mg} / \mathrm{kg}($ Ext 2) $\mathrm{BW}$ on globulin (A), blood glucose (B), phosphorous (C), total bilirubin (D) and total protein levels in normal and diabetic male Wistar rats. Each column represents mean $\pm \mathrm{SD}(\mathrm{n}=3)$. Group 1: diabetic + Ext 1; Group 2: diabetic + Ext 2; Group 3: diabetic + metformin; Group 4: diabetic control; Group 5: normal + Ext 1; Group 6 normal + Ext 2; Group 7: normal control; Group 8: normal + 1\% Tween 80 .

activity of other studied medicinal plants to their ability to restore the function of pancreatic tissue, by causing an increase in the insulin output or by a decrease in the intestinal absorption of glucose (Ibeh and Ezeaja, 2011; Neelesh et al., 2010). Biomarker enzymes such as alkaline phosphatase and alanine aminotransferase may be used for the evaluation of hepatic disorders (Ghandi et al., 2012). This may be substantiated by the ALKP results in Figure 1 depicting elevated ALKP levels found in the STZ-induced diabetic groups (Groups 1 to 4). A significant reduction in the activity of ALKP in $B$. discolor treated diabetic rats indicates a potential hepatoprotective 


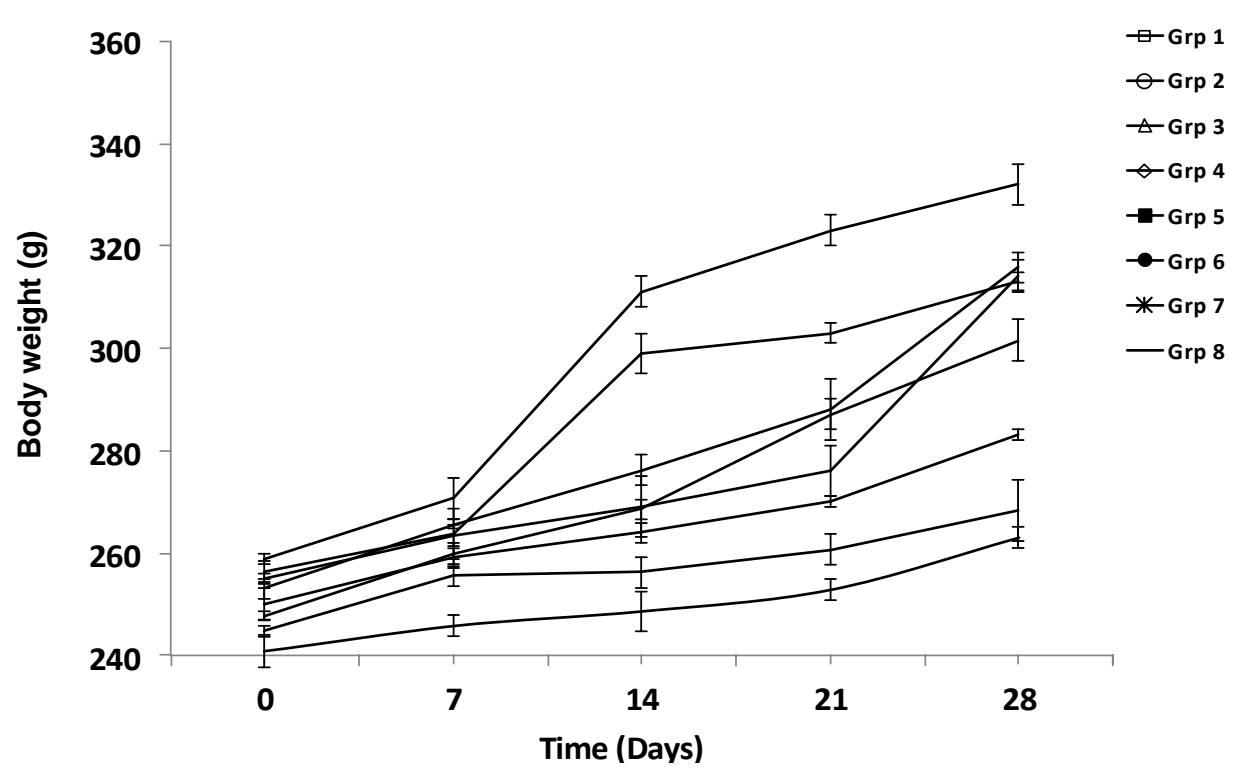

Figure 3. Body weight gains in the different animal groups during the 28 day experimental period. Data is expressed as a mean of $n=4$. Group 1: Diabetic + Ext 1; Group 2: diabetic + Ext 2; Group 3: diabetic + metformin; Group 4: diabetic control; Group 5: normal + Ext 1; Group 6: normal + Ext 2; Group 7: normal control; Group 8: normal + 1\% Tween 80.

role in preventing possible diabetic complications. The elevation of urea, creatinine and total proteins may be considered as a significant marker for renal dysfunction (Carlo et al., 1996). Results in Figure 1 show a decrease in the urea levels of animals treated with $B$. discolor extract. The increase in urea levels for the STZ-induced control rats (Group 4) and the STZ-induced rats receiving the reference drug (Group 3) may be due to the high protein present in B. discolor (Haschick and Kerley, 1997). Blood urea nitrogen is a metabolite produced from dietary protein (Al Faris et al., 2011). Hyperglycaemia results in the generation of free radicals which may exhaust antioxidant defenses, thereby leading to the disruption of cellular functions and oxidative damage to membranes (Dangi and Mishra, 2011). Decreased protein levels may be attributed to the antioxidant activity of organic compounds within the $B$. discolor extract which may play a role in reducing the protein levels as can be seen in Figure 2 (Al Faris et al., 2011). Creatinine levels for STZ-induced rats receiving the $B$. discolor extract and those receiving the reference drug (metformin) all had creatinine levels which were within the normal range when compared with the STZ-induced control group whose levels remained below the acceptable minimum range. By the end of the 28 days treatment period, the serum levels for ALB, Amyl, ALT, Ca, CHOL, GLOB, P, TBIL and TP were not significantly different in comparison to the control group. The induction of diabetes by STZ has been associated with a loss in BW due to increased muscle wasting and loss of tissue protein (Chatterjee and Shinde, 2002; Gupta et al., 2012). The use of $B$. discolor at the $600 \mathrm{mg} / \mathrm{kg} \mathrm{BW}$ dose improved the
BW in diabetic rats over the 28 days study period. This increase in the BW of diabetic rats may be attributed to an improvement in insulin secretion and glycemic control (Eliza et al., 2009; Genet et al., 1999). It has also been reported by Rajkumar et al. (1997) that increased anabolic reactions may be responsible for weight gain in diabetic rats.

Conclusively, the results of this study have demonstrated that $B$. discolor has significant antidiabetic potential and may be acting through the restoration of pancreatic tissue, stimulation of $\beta$-cells or by decreasing intestinal glucose absorption. Further studies are required to isolate and characterize antidiabetic bioactive compounds, and establish the mechanism(s) of action.

\section{ACKNOWLEDGEMENTS}

This work was supported by a grant from the National Research Foundation (NRF), South Africa and the Durban University of Technology.

\section{REFERENCES}

Al Faris N, Al Othman ZA, Ahmad D (2011). Effects of Messembrrybryanthemum forsskalei Hochst seeds in lowering glucose/lipid profile in streptozotocin-induced diabetic rats. J. Food Sci. Technol. 48: 616-621.

Carlo F, Welters N, Corneils H, Petr B (1996). Enhance renal vein ammoniaefflux after protein meal in the pig. J.Hepatol. 31: 489-496

Chatterjee M, Shinde R (2002). Textbook of Medical Biochemistry. Jaypee Brothers Medical Publishers, New Delhi. India.

Colagiuri R, Colagiuri S, Yach D (2006). The Answer to Diabetes Prevention: Science, Surgery, Service Delivery, or Social Policy?. 
Am. J. Pub. Health. 96:1-8.

Dangi K, Mishra S (2011). Antioxidative and $\beta$-cell regeneration effect of Capparis aphylla stem extract in streptozotocin induced diabetic rats. Biol. Med. 3: 82-91.

Eliza J, Daisy P, Ignacimuthu S, Duraipandiyan V (2009). Antidiabetic and antilipidemic effect of eremanthin from Costus speciosus (Koen.)Sm. in STZ-induced diabetic rats. Chemico-Biolog. Interact. 182: $67-72$.

Fennell C, Lindsey K, McGaw L, Sparg S, Stafford G, Elgorashi E, Grace O, Van Staden J (2004). Assessing African medicinal plants for efficacy and safety: pharmacological screening and toxicology. J. Ethnopharmacol. 94: 205-217.

Genet S, Raosaheb K, Najma Z (1999). Effects of vanadate, insulin and fenugreek (Trigonella foenum graecum) on creatine kinase level in tissues of diabetic rat. Ind. J. Exp. Biol. 37: 200-202.

Ghandi G, Ignacimuthu S, Paulraj M (2012). Hypoglycemic and $\beta$-cells regenerative effects of Aegle marmelos (L.) Corr. bark extract in streptozotocin-induced diabetic rats. Food Chem. Toxicol. 50: 16671674.

Grover J, Yadav S, Vats V (2002). Medicinal plants of India with antidiabetic potential. J. Ethnopharmacol. 81: 81-100.

Gupta R, Kumar D, Chaudhary A, Maithani M, Singh R (2012). Antidiabetic activity of Passiflora incarnat Linn. in streptozotocin induced diabetes in mice. J. Ethnopharmacol. 139: 801-806.

Haschick S, Kerley G (1997). Factors influencing forage prefernce of bushbuck and boer goats for Subtropical Thicket plants. Afr.J. Range Forage Sci. 14: 49-55.

Holt R (2004). Diagnosis, epidemiology and pathogenesis of diabetes mellitus: an update for psychiatrists. British J.Psychiatry. 184: s55s63.

Hutchings A (1996). Zulu Medicinal Plants: An Inventory, University of Natal press, KZN, pp $316-317$.

Ibeh B, Ezeaja M (2011). Preliminary study of anti-diabetic activity of the methanolic leaf extract of Axonopus compressus (P. Beauv) in alloxin-induced diabetic rats. J. Ethnopharmacol. 138: 713-716.

Iwueke A, Nwodo F (2008). Antihyperglycaemic effect of aqueous extract of Daniella oliveri and Sarcocephalus latifolius roots on key carbohydrate metabolic enzymes and glycogen in experimental diabetes. Biokemistri. 20:63-70.

Jeremy C, Whiteman M (2003). Antioxidant activity of some Chinese herbs. Free Radical Biol. Med. 26: 1231-1237.

Kamgang R, Mboumi R, Fondjo A, Tagne M, N'dillè G, Yonkeu J (2008). Antihyperglycaemic potential of the water-ethanol extract of Kalanchoe crenata (Crassulaceae). J. Natur. Med. 62: 34-40.
Moller D (2001). New drug targets for type 2 diabetes and the metabolic syndrome. Nature. 414: 821-827.

Musabayane C, Mahlalela N, Shode F, Ojewole J (2005). Effects of Syzygium cordatum (Hochst.) [Myrtaceae] leaf extract on plasma glucose and hepatic glycogen in streptozotocin-induced diabetic rats. J. Ethnopharmacol. 97: 485-490.

Neelesh M, Sanjay J, Sappa M (2010). Antidiabetic potential of mdeicinal plants. Acta Poliniae Pharmaceutica - Drug Res. 67: 113118.

Odhav B, Kandasamy T, Khumalo N, Baijnath H (2010). Screening of African traditional vegetables for their alpha-amylase inhibitory effect. J. Med. Plants Res. 4: 1502-1507.

Rajkumar L, Srinivasan N, Balasubramanian K, Govindarajulu P (1997). Increased degradation of dermal collagen in diabetic rats. Ind. J. Exp. Biol. 1081-1083.

Rheeder P (2006). Type 2 diabetes: The emerging epidemic. SA Fam Pract. 48: 20 .

Tonye Mahop M, Mayet M (2007). En route to biopiracy? Ethnobotanical research on anti diabetic medicinal plants in the Eastern Cape Province South Africa. Afr. J. Biotechnol. 6: 2945-2952.

Van Wyk B, van Wyk P (2007). How to Identify Trees in Southern Africa. Struik Publishers, Cape Town.

$\mathrm{WHO}$ ( 2002). Launches of the first global strategy on the traditional medicine. WHO Press release.

Wild S, Roglic G, Green A, Sicree R, King H (2004). Global Prevalence of Diabetes: Estimates for the year 2000 and projections for 2030. Diabetes Care. 27: 1047-1053. 